\title{
Erratum to the paper \\ ON CLASSICAL STABILITY CONDITIONS FOR A SPINNING SHELL
}

Quarterly of Applied Mathematics XXVII, 247-254

The authors of this paper are J. P. Sharma, P. C. Rath and Daulat Ram (not, as was previously given, P. C. Rath and Daulat Ram).

\section{Errata to the paper}

\section{CHARACTERIZATION OF SINGLE-BLOW TEMPERATURE \\ RESPONSES BY FIRST MOMENTS}

BY

GERHARD F. KOHLMAYR

Quarterly of Applied Mathematics XXVII, 162-172

In the first line following Eq. (10) on p. 163 replace $\mathscr{L}\left\{\Xi_{1}\left(-a^{2} t\right) e^{-a t} ; s\right\}$ by $\mathscr{L}\left\{a^{2} \Xi_{1}\right.$ $\left.\left(-a^{2} t\right) e^{-a t} ; s\right\}$.

The right-hand side of Eq. (11) on p. 163, $e^{-a}[g(t)-1]+a^{2} \int_{0}^{t} \Xi_{1}\left(-a^{2} \theta\right) e^{-a \theta} d \theta$, should be replaced by $e^{-a}[g(t)-1]+1-a^{2} \int_{0}^{t} \Xi_{1}\left(-a^{2} \theta\right) e^{-a \theta} d \theta$.

In the twelfth line from the top on p. 164 replace $\left\{\int_{0}^{\infty}\left[G_{\text {exp }}(t)-G(a, t) d t\right\}^{1 / 2}\right.$ by $\left\{\int_{0}^{\infty}\left[G_{\text {oxp }}(t)-G(a, t)\right]^{2} d t\right\}^{1 / 2}$. 